\title{
Sitting at Patients' Bedsides May Improve Patients' Perceptions of Physician Communication Skills
}

\author{
Susan E. Merel, MD*, Christy M. McKinney, PhD, MPH' ${ }^{1,2}$, Patrick Ufkes, BA, BS ${ }^{3}$, Alan C. Kwan, MD³, Andrew A. White, MD ${ }^{1}$
}

${ }^{1}$ Division of General Internal Medicine, Department of Medicine, University of Washington, Seattle, Washington; ${ }^{2}$ Department of Oral Health Sciences, University of Washington, Seattle, Washington; ${ }^{3}$ Department of Medicine, Johns Hopkins University, Baltimore, Maryland.

Sitting at a patient's bedside in the inpatient setting is recommended as a best practice but has not been widely adopted. Previous studies suggest that a physician's seated posture may increase the patient's perception of time spent in the room but have not included hospitalists. We performed a cluster-randomized trial of seated versus standing physician posture during inpatient rounds on a hospitalist service at an academic medical center. Patients whose physician sat were significantly more likely to rate their physician highly on measures of listening carefully and explaining things in a way that was easy to understand. The average time spent in the patient's room was approximately 12 minutes and was not affected by physician posture. Patients' perception of the time their physician spent in their room was not affected by physician posture. Sitting at the bedside during rounds does not increase the amount of time spent with the patient but may improve patient-physician communication. Journal of Hospital Medicine 2016;11:865-868. (c) 2016 Society of Hospital Medicine
Sitting while interacting with patients is standard in the outpatient setting and encouraged in the inpatient setting as a best practice. ${ }^{1,2}$ Michael W. Kahn defined etiquette-based medicine as a set of easily taught behaviors that demonstrate respect for the patient; sitting at the bedside is included. ${ }^{1}$ A prominent healthcare consulting group also recommends that physicians and nurses sit at the bedside, claiming that "the patient will estimate you were in the room 3 times longer." 3 Previous studies suggest patients may perceive physicians who sit at the bedside as more compassionate and as spending more time with them, and may perceive the overall interaction as more positive when the physician sits. ${ }^{4-6}$ Two small studies found that patients perceived the physician as having spent more time with them if he or she sat rather than stood. ${ }^{5,6} \mathrm{~A}$ study in the emergency department found no effect of posture on patient perception of physician communication skills, and a study of a single attending neurosurgeon found that patients reported a better understanding of their condition when the physician sat. ${ }^{5,6}$ The effect of physician posture on hospitalist physician-patient communication has not been previously studied. Despite evidence that sitting in the inpatient setting may improve physician-patient communication, studies suggest that physicians rarely sit at the bedside of inpatients. ${ }^{7,8}$

\footnotetext{
*Address for correspondence and reprint requests: Susan E. Merel, MD, 1959 NE Pacific Street, Box 356429, Seattle, WA 98195-6429; Telephone: 206-616-4088; Fax: 206-221-8732; E-mail: smerel@uw.edu

Additional Supporting Information may be found in the online version of this article.

Received: March 14, 2016; Revised: May 23, 2016; Accepted: May 31, 2016

2016 Society of Hospital Medicine DOI 10.1002/jhm.2634

Published online in Wiley Online Library (Wileyonlinelibrary.com).
}

We conducted a cluster-randomized trial of the impact of hospitalist physician posture during morning rounds. We hypothesized that patients whose physician sat rather than stood would perceive that their physician spent more time with them and would rate the physician's communication skills more highly. We also hypothesized that sitting would not prolong the length of the patient-physician encounter.

\section{PATIENTS AND METHODS}

We conducted a cluster-randomized clinical trial with a crossover component randomizing physicians on the order of sit/stand within a consecutive 7-day workweek. We enrolled patients being cared for by attending hospitalists on a resident-uncovered general internal medicine service in an academic tertiary care hospital. We also enrolled the hospitalists and collected demographics and practice information. Wall-mounted folding chairs (Figure 1) were installed in all rooms on two 28-bed units for use by physicians. Eligible patients were newly admitted or transferred from the intensive care unit between June 2014 and June 2015, English speaking, and adults who consented to their own medical care. Physicians were randomly assigned to sit or stand during morning rounds for the first 3 days of their workweek. The last 4 days they provided care using the other posture. Blocks of 4 weeks were used to randomize the sit/stand order.

We measured the length of the physician-patient interaction, asked both the physician and the patient to estimate the length of the interaction, and administered a written survey to the patient with questions about the physician's communication skills. Research assistants timed the interaction from outside the room and entered the room to consent patients and administer the survey after the physician departed. Survey questions were modeled on the physician 
communication questions from the Hospital Consumer Assessment of Healthcare Providers and Systems (HCAHPS) survey. We aggregated all answers other than the most positive answer because HCAHPS questions are analyzed according to a "top box" methodology. Adherence to the intervention was measured by asking the physician whether he or she actually sat or stood for each interaction. We administered a survey to physicians to collect demographics and feedback.

We estimated descriptive statistics for physician and patient participants using cross-tabs and means. To estimate associations, we used logistic and linear regression that employed cluster-adjusted $t$ statistics and clustered patients within providers. This method optimizes estimation of standard errors (and corresponding confidence intervals and $P$ values) when the number of clusters is small (16 physicians). ${ }^{9}$ For our primary analysis, we analyzed as randomized using an intent-to-treat approach. In other words, those assigned to the standing group were analyzed in the standing group even if they actually sat (and vice versa). In a sensitivity analysis we used the same methods to analyze the data according actual provider

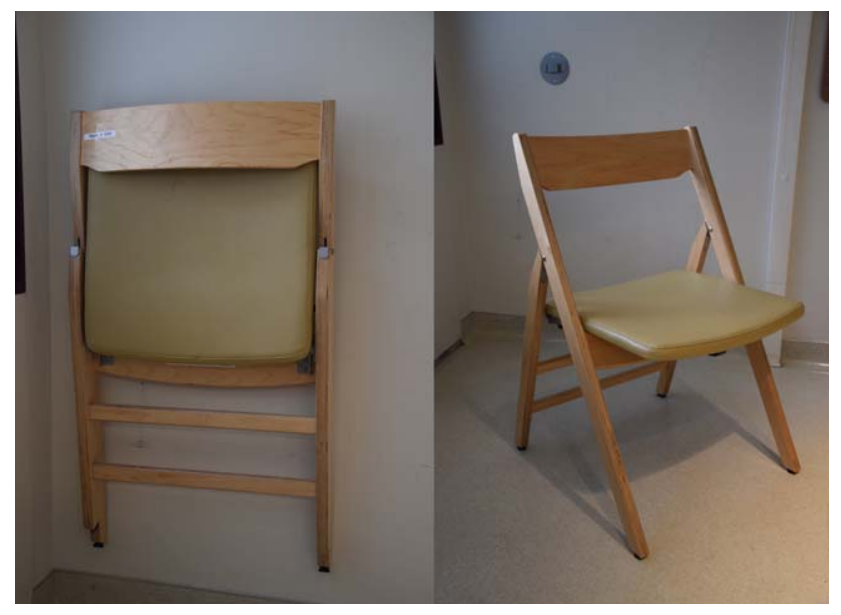

FIG. 1. Chair used in the study. posture as reported by the physician and not as randomized. We calculated the mean and range of the number of patients seen by physicians. We compared estimates of time spent between patients and providers and patients' satisfaction according to provider posture. We complied with the Consolidated Standards of Reporting Trials 2010 guidelines. ${ }^{10}$ Our institutional review board approved this project. All participants provided written consent.

\section{RESULTS}

All 17 hospitalists attending on the service consented to participate; 1 did not see any patients involved in the study and was removed from the analysis. Sixtynine percent were female and $81 \%$ had been in practice for 3 years or less at the time of study enrollment; $94 \%$ reported standing when assigned to stand and $83 \%$ reported sitting when assigned to sit. We found $31 \%$ of physicians reported they routinely sat before participating in the study, and $81 \%$ said they would sit more after the study; this result approached statistical significance (exact McNemar $P=0.06$ ). Of the 11 physicians who reported not routinely sitting before the study all, 7 cited not having a place to sit as a reason for not sitting. Other rationale provided included being too short to see the patient if seated, believing rounds would take more time if seated, and concerns about contact precautions. Comments in the postintervention survey regarding why providers planned to sit more centered around themes of having chairs available, thinking that sitting improves communication, and thinking that patients prefer providers to sit.

Two hundred eleven patients were assessed for eligibility. Fifty-two were excluded ( 27 did not meet inclusion criteria and 25 declined to participate), leaving 159 participating patients. Seven patient-physician pairs were inadvertently assigned the wrong intervention but were analyzed as randomized. There were no demographic differences between patient groups (Table 1). Physicians participating in the study saw an average of 13 study patients (range, 1-18) during the study. Mean time spent in the patient's room during

TABLE 1. Patient Characteristics

\begin{tabular}{|c|c|c|c|c|c|}
\hline & \multicolumn{2}{|c|}{$\begin{array}{l}\text { Patients Seen by Seated } \\
\text { Physician, } N=66\end{array}$} & \multicolumn{2}{|c|}{$\begin{array}{l}\text { Patients Seen by Standing } \\
\text { Physician, } N=93\end{array}$} & \multirow[b]{2}{*}{$P$ Value } \\
\hline & $n$ & $\%$ & $n$ & $\%$ & \\
\hline \multicolumn{6}{|l|}{ Patient age, y } \\
\hline $18-39$ & 16 & 25.4 & 25 & 27.5 & 0.59 \\
\hline $40-59$ & 17 & 27.0 & 30 & 33.0 & \\
\hline $60+$ & 30 & 47.6 & 36 & 39.6 & \\
\hline \multicolumn{6}{|l|}{ Gender } \\
\hline Male & 32 & 49.2 & 43 & 46.2 & 0.71 \\
\hline Female & 33 & 50.8 & 50 & 53.8 & \\
\hline \multicolumn{6}{|l|}{ Ethnicity } \\
\hline Caucasian & 54 & 84.4 & 67 & 73.6 & 0.24 \\
\hline Asian or Pacific Islander & 3 & 4.7 & 5 & 5.5 & \\
\hline Other & 7 & 10.9 & 19 & 20.9 & \\
\hline
\end{tabular}


TABLE 2. Patient Perceptions of Physician Communication

\begin{tabular}{|c|c|c|c|c|c|}
\hline & \multicolumn{2}{|c|}{$\begin{array}{l}\text { Patients Seen by Seated Physi- } \\
\qquad \text { cian, } N=66\end{array}$} & \multicolumn{2}{|c|}{$\begin{array}{l}\text { Patients Seen by Standing } \\
\text { Physician, } \mathrm{N}=93\end{array}$} & \multirow[b]{2}{*}{$P$ Value } \\
\hline & $\mathrm{n}$ & $\%$ & $\mathrm{n}$ & $\%$ & \\
\hline \multicolumn{6}{|c|}{ Patient perception of physician communication on that day's rounds } \\
\hline \multicolumn{6}{|c|}{ Today on rounds, how often did this physician.... } \\
\hline \multicolumn{6}{|c|}{ Explain things in a way that was easy to understand? } \\
\hline Never, sometimes, or usually & 7 & 10.9 & 22 & 23.9 & 0.05 \\
\hline Always & 57 & 89.1 & 71 & 76.1 & \\
\hline \multicolumn{6}{|l|}{ Listen carefully to you? } \\
\hline Never, sometimes, or usually & 4 & 6.1 & 19 & 20.4 & 0.02 \\
\hline Always & 62 & 93.4 & 74 & 79.6 & \\
\hline \multicolumn{6}{|l|}{ Interrupt you when you were talking? } \\
\hline Always, sometimes, or usually & 4 & 6.5 & 9 & 10 & 0.46 \\
\hline Never & 58 & 93.6 & 81 & 90 & \\
\hline \multicolumn{6}{|l|}{ Treat you with courtesy and respect? } \\
\hline Never, sometimes, or usually & 0 & 0 & 7 & 7.6 & Not estimable \\
\hline Always & 63 & 100 & 85 & 92.4 & \\
\hline \multicolumn{6}{|c|}{ Please rate the amount of time this physician spent with you today during morning rounds. } \\
\hline Too little & 1 & 1.6 & 3 & 3.5 & 0.41 \\
\hline Just right & 63 & 98.4 & 84 & 96.5 & \\
\hline \multicolumn{6}{|c|}{ Did you have any important questions or concerns about your care that you did not bring up with this doctor today?* } \\
\hline Yes & 4 & 6.6 & 9 & 10.3 & 0.26 \\
\hline № & 57 & 94.4 & 78 & 89.7 & \\
\hline
\end{tabular}

NOTE: All variables missing $<5 \%$. "Missing $6.9 \%$.

rounds was 12:00 minutes for seated physicians and 12:10 for standing physicians $(P=0.84)$. Regardless of provider posture, patients overestimated the amount of time their physician spent in the room (mean difference 4:10 minutes, $P=0.01$ ). Patients' estimates of the time the physician spent did not vary by posture (16:00 minutes for seated, $16: 19$ for standing, $P=0.86)$.

Patients whose physician sat on rounds were statistically significantly more likely to choose the answer "always" to the questions regarding their physician listening carefully to them $(P=0.02)$ and explaining things in a way that was easy to understand $(P=$ 0.05 , Table 2). There was no difference in the patients' response to questions about the physician interrupting the patient when talking or treating them with courtesy and respect. Nearly all patients chose "just right" when asked to rate the amount of time their physician had spent with them on rounds (Table 2 ). The results of our sensitivity analysis that classified physicians according to their actual posture yielded different results; none of the findings in that analysis including questions regarding the physician listening carefully or explaining things in a way that was easy to understand were statistically significant (see Supporting Information, Appendix 1, in the online version of this article).

\section{DISCUSSION}

In our study involving general medicine inpatients cared for by academic hospitalists, physicians did not spend more time in the room when seated, and were willing to adopt this practice. Patients perceived that seated compared to standing physicians listened more carefully and explained things in a way that was easy to understand when analyzed using an intent-to-treat approach. Patients did not perceive that seated physicians spent more time with them than standing physicians. To our knowledge, this is the first study showing the effects of hospitalist rounding posture on patient experience.

Our finding that patients rated seated physicians more highly on listening carefully and explaining things well indicates that training hospitalists to sit at the bedside may ultimately improve patient satisfaction. Our findings suggest seated interaction may improve satisfaction with communication without increasing time burden on physicians. However, given that these findings were not statistically significant when we analyzed our data according to actual behavior, larger studies should verify the impact of physician posture on patient experience.

Previous studies found that a minority of physicians sit in the inpatient setting, but did not study barriers to sitting while on rounds. ${ }^{7,8}$ A majority of physicians in our study sat when instructed to do so and when chairs were provided, and over $80 \%$ of physicians in our study said they planned to continue sitting while on rounds after the study was complete. A lack of chairs may be a major barrier to physicians adopting this facet of etiquette-based medicine, and institutions wishing to promote this practice should consider providing chairs. Written comments from physician participants suggest physicians who are introduced to this 
practice enjoy sitting and think it improves physicianpatient communication. Further studies are needed to test our assumption that physicians continue sitting when chairs are provided.

Our work differs from previous studies. Johnson et al. studied interactions in the emergency room with a mean length of 8.6 minutes, ${ }^{5}$ and Swayden et al. studied postoperative visits by a single neurosurgeon with a mean length of about 1 minute. ${ }^{6}$ One explanation for the lack of a difference in time spent by posture might be that an average visit time of 12 minutes passes a threshold where patients make more accurate estimates of visit length or where factors other than posture more strongly influence perceptions of duration.

Limitations of our study include the relatively small sample size, single location, and limitation to Englishspeaking patients able to consent themselves. Reasons for the limited sample size include that chairs were only installed in 2 units, and not all patients on the unit were under the care of participating physicians. Physician subjects were not blinded to their interactions being timed or to the fact that patients were surveyed about their communication skills. It is possible that factors that may have affected patients' responses such as severity of illness, number of consultants involved in their care, or prior experiences in the healthcare system were not equally distributed between our 2 groups. Additionally, our use of questions similar to those used in the HCAHPS instrument is not compliant with Centers for Medicare and Medicaid Services (CMS) policy. We caution others against using questions that might invalidate their hospital's participation in CMS payment programs.

Our study was limited to rounds involving 1 physician; our practice is that in a larger team the presenting member is encouraged to sit and others sit if there are additional chairs. Best practices on a teaching service are unclear and could be the subject of further study. The longer-term sustainability of the practice of sitting on rounds is unclear. However, our physician subjects reported that they plan to continue to sit after the study, and we have shared the results with physicians in order to provide them with evidence supporting this practice. Not having a place to sit and thinking that sitting increases the amount of time spent on rounds were concerns provided in our preintervention survey, and we believe our study addresses these concerns.

Our study demonstrates the effects of a simple intervention on patient satisfaction without increasing burden on providers. Sitting at the bedside does not impact the amount of time spent with the patient, but may improve the patient's perception of the physician's communication skills and thus impact the patient experience. This simple intervention could improve patient satisfaction at little cost.

\section{Acknowledgements}

The authors acknowledge Tom Staiger, MD, UWMC Medical Director, for his assistance with obtaining chairs for this study.

Disclosure: Nothing to report.

\section{References}

1. Kahn M. Etiquette-based medicine. N Engl J Med. 2008;358(19): 1988-1989.

2. Sorenson E, Malakouti M, Brown G, Koo J. Enhancing patient satisfaction in dermatology. Am J Clin Dermatol. 2015;16:1-4.

3. The Studer Group. Q\&A: Getting-and keeping-physicians on board: best practices. Available at: https://www.studergroup.com/ hardwired-results/hardwired-results-07/qa-get-physicians-on-boardlearn-best-practices. Accessed February 29, 2016.

4. Bruera E, Palmer JL, Pace E, Zhang K, Willey J, Strasser F, Bennett MI. A randomized, controlled trial of physician posture when breaking bad news to cancer patients. Palliat Med. 2007;21:501-505.

5. Johnson RL, Sadosty AT, Weaver AL, Goyal DG. To sit or not to sit? Ann Emerg Med. 2008;51:188-193.

6. Swayden KJ, Anderson KK, Connelly LM, Moran JS, McMahon JK, Arnold PM. Effect of sitting vs. standing on perception of provider time at bedside: a pilot study. Patient Educ Couns. 2012;86(2):166-171.

7. Tackett S, Tad-y D, Rios R, Kisuule F, Wright S. Appraising the Practice of Etiquette-Based Medicine in the Inpatient Setting. J Gen Intern Med. 2013;28(7):908-913.

8. BlockL, Hutzler L, Habicht R, et al. Do internal medicine interns practice etiquette-based communication? A critical look at the inpatient encounter. J Hosp Med. 2013; 8:631-634.

9. Esarey J, Menger A. Practical and effective approaches to dealing with clustered data [unpublished manuscript]. Department of Political Science, Rice University, Houston, TX. Available at: http://jee3.web.rice. edu/cluster-paper.pdf. Accessed February 29, 2016.

10. Schulz KF, Altman DG, Moher D. Consort 2010 statement: updated guidelines for reporting parallel group randomized trials. Ann Intern Med. 2010;152(11):726-732. 